\title{
A new modular electronics approach applied to instrumentation units
}

\author{
Ghislain Guerrero ${ }^{1}$, Valentin Chomel ${ }^{1}$, Floriane Monteil ${ }^{2}$, Olivier Pinto ${ }^{1}$ \\ ${ }^{1}$ Safran Data Systems, 5, avenue des Andes - 91978 Courtaboeuf Cedex, France \\ ${ }^{2}$ Safran Data Systems GmbH, Friedrich-Ebert-Str. 51429 Bergisch Gladbach, Germany \\ ghislain.guerrero@safrangroup.com
}

\begin{abstract}
One of the main challenges of an instrumentation engineer is to provide accurately the required information while preserving the test vehicle inherent behavior which, somewhere, breaches the universal Heisenberg uncertainty principle.

This article presents the results of a technology investigation based on an original modular $3 \mathrm{D}$ electronic design. This technology aims at minimizing the intrusiveness of an instrumentation system while offering flexibility, scalability, reliability and performances.
\end{abstract}

Based on a return of experience from the legacy and current instrumentation systems, the study also addresses the daily operational constraints faced by an instrumentation engineer: auto-configuration, plug and play, fast reconfiguration, harsh environmental conditions, ...

Key words: FTI - Intrusiveness - SWAP - 3D electronics - IOT

\section{Background}

Flight Test Instrumentation is at the heart of several challenges. It requires collecting the most precise and reliable data with minimal influence to the vehicle being tested. It must minimize intrusiveness while maximizing data quality. In addition, it is used at a critical time, just before market introduction and mass production. The financial stakes are high. To allow for fast delivery, it must therefore also be reliable, flexible and quick to set up. This is essential in order to enter the market and refund R\&D investments as quickly as possible. This instrumentation must also be scalable to adapt to all cases (from a few measurements to tens of thousands in the same test vehicle among commercial or military aircrafts, fighters, helicopters, space launch vehicles, armaments, maritime vehicles, automotive,...). It must also be dismantled without leaving signs after tests or a certification campaign. The instrumentation of a production vehicle does not allow neither drilling of mounting holes nor the use of mods but must allow fast and efficient troubleshooting. In real life, the complex process of validation and certification of an aircraft is very precise and perfectly mastered by aircraft manufacturers, but a test campaign is also full of unknowns which force the test engineers to react to unexpected events and therefore the instrumentation team has to adapt to new requirements, as quickly and efficiently as possible.

Legacy instrumentation systems are not natively suited for all these use-cases. They require compromises, modifications, time and work to get results that are not always satisfactory.

\section{Global trend in Flight Test Instrumentation}

Instrumenting an aircraft to successfully succeed in getting flight certification is not an easy task. It is a comprehensive area of expertise that has pushed each product generation to adapt, improve and take advantage of new technologies to achieve maximum efficiency in flight test campaigns. However, we must approach these changes and system improvements with the choices inherited from the past in mind.

Flight Test Instrumentation, in its mission to ensure valuable flight test results, was initially led by the safety of data storage and the first needs of specific physical value acquisition. Since then, the main challenges are to get proper data to record, display and transmit to ground stations. The measured data must also be accurately synchronized on a common time base. It enables the engineers to process them at the test vehicle scale and understand complex physical effects that are affecting the behavior of the vehicle.

Historically, instrumentation systems used to be centralized, installed in cabin (e.g. 19-inch 
racks) [1]. This architecture centralized the entire amount of data. Such systems ensured accurately timestamped synchronous data acquisitions.

These systems, sole hearts of the instrumentation, then combined all needed operations in one location: they could acquire, process, record, stream out and telemeter from one unit, therefore offering a reliable solution. The different applications requiring very specific features were not left aside: customization was made simple because of the centralization. Although natively designed to fit the FTI requirements, the centralized systems showed limitations. One unit gathering all data means several connections through the vehicle to the data sources: sensors, buses, cameras... and all the constraints that follow. For example, the interferences on cables corrupting data could make transmissions erroneous (bit swapping). In addition to the data corruption risks, the space available in the vehicle is limited, and so is the room left for cables.

The need to reduce the number of flights necessary for a test, to maintain or even improve the level of performance of acquisitions, the reduction of electronics footprint and the emergence of new processing capacities (on board or on the ground) with "BigData" has triggered the possibility of an evolution, going in the direction of decentralization, more data, more flexibility, more precision and multiple optimizations.

Over the past 15 years, instrumentation systems have become more decentralized. The advantages are for example better data accuracy, fewer cables, less weight, less impact on the tested vehicle. New modular equipment has come into operation, able to adapt to many specific applications in aircrafts, rotorcrafts, UAV, but also missiles and launch vehicles.

From sensors to telemetry, new standards, methods and concepts emerged. Network architectures have become more distributed and very efficient using standards such as Ethernet for fast data transfers, without compromising quality, even over long distances. Today, there is no longer a central unit but remote devices smartly spread in the aircraft, located in the few "empty spaces". Reducing the distance from the sensor to the signal conditioning and acquisition system improved the quality of the data. It can be digitalized as soon as acquired, in a much higher quality thanks to improvements regarding bandwidth and storage capacity. With modularity inside high performance small units, those distributed architectures address a lot of use-case specific requirements.

Wireless protocols can also be used for more flexibility (for the instrumentation of a helicopter rotor for example). [2]

However, those systems are still aggregators of acquisition, processing and communication capacities. They are more distributed than the previous generation but they still have a significant impact on the vehicle linked to their installation and operation. This paper presents the thoughts carried out on the future of instrumentation as well as its results.

\section{The Internet of Things (IoT) Trend}

The Internet of Things (IOT) is a system of interdependent devices connected to a network and/or to one another, exchanging data (autonomously in general). Basically, the loT is a collection of electronic devices that can share information among themselves.

From "ubiquitous computing" in the 1990's to the emergence of Industrial loT (Ilot), the loT market grew exponentially from zero to several trillion dollars. Electronics have considerably reduced in size (down to being smaller than connectors in some cases), energy consumption has drastically decreased enabling life-time of years on batteries, networks have become wireless and more and more efficient, embedded or cloud computing capabilities followed Moore's law.

The information value loop (Use of sensors to generate information, transfer and collection of this information, discernment of patterns or relationships between phenomena, enabling descriptions, predictions, or prescriptions for action leading to initiating, maintaining, or changing an event or a physical state) [3] has found applications in all areas of industry and life (Examples include smart factories, smart home devices, medical surveillance devices, portable fitness trackers, smart city infrastructure, vehicle telematics, among lots of others) to the point that today there are 5 times more loT devices in the world than humans (and still a two digit growth).

\section{Limits of the current modular instrumentation electronics}

As previously discussed, an instrumentation device needs to be scalable and modular in order to offer the best size, weight and power (SWAP) efficiency. In this respect two main categories of mechanical architecture exist on the market: the racks and the stacks. Both offers advantages but also drawbacks: 
- Racks: quick access to each module but fixed size with step effect when switching from a size to another (e.g. from 9 slots to 16 slots). Usually less ruggedized and compact than stacks.

- Stacks: scalable size defined by the exact number of module needed by the user but changing a module requires opening the stack. Most of the time, more ruggedized and more compact than racks

In both cases, the modules used for the racks and the stacks are based on a printed board circuit (PCB), an external connector and one (racks) or two (stacks) internal connectors. Both designs lead to parallelepiped volumes which hit certain limits when downsized:

For interconnection between modules, the design engineer is facing a challenging alternative:

- Apply the downsizing to internal connectors with very tiny, small pitch and low profile connectors, as the one used today in most of our mobile devices, that are proven solution for high speed communication. However, these kinds of connectors have also shown their limits in terms of ruggedization and usually do not cope with:

- Repetitive assembly and disassembly by the end-user who needs even sometimes to work directly on the field

- The mechanical harsh environments frequently encountered in flight testing where overall vibrations and transient shocks levels can be very harsh / severe.

- Do not apply the downsizing to the internal connectors and keep legacy flight proven part numbers. In this case the volume ratio between the interconnection items and the electronic parts hosting the features becomes too big

For the external connectors, there are traditionally two kinds of connectors: The ones used to connect the signal/data source (sensors, voltage lines, communication buses, ...): the "D-type" and the cylindrical connectors.

The " $D$ type" connectors, with the famous SubD type connector, are the natural choice, whatever the stack or rack design. They offer the best profile in terms of pin count when mounted at the extremity of a PCB. The downscaling of devices leads the connector manufacturer to propose higher density Sub-D $H D$ and miniature versions: micro-D and even nano-D connectors. The main drawback of these connectors comes from the fact that, at a certain point, they do not offer anymore the minimal wire gauge \#24 usually asked by the instrumentation engineers and also no more crimpable contact and only pigtail type connection that prevents from any last minute modification of the harness which is a key asset in flight test domain.

The cylindrical connectors, as the famous 38999 series, are the reference connectors in aerospace domain to establish connection between devices. However, for modular systems, this kind of connectors mostly requires intermediate backpanels that routes the signals from the flat PCB based modules to the connectors, affecting badly the scalability, the versatility and the overall size of the instrumentation device

\section{Leveraging the $3^{\text {rd }}$ dimension}

More and more electronic technology offer the capability to take benefits of the $3^{\text {rd }}$ dimension in order to optimize integration factor, placing more functions in a given space while optimizing the distance between the electronic components. 3D electronic integration can take place at different level:

At PCB level: rigid/flex technology allow to fold in 3 dimensions a flat PCB composed of rigid parts where the components are mounted and flexible ones insuring the interconnections between the various rigid ones. This technology offers the possibility of a secured connection of device components with the assurance of polarity and contact stability, as well as a reduction in plug and connector components [4]. The rigid/flex technology exists at various scales and complexity levels (number and nature of layers stacked together).

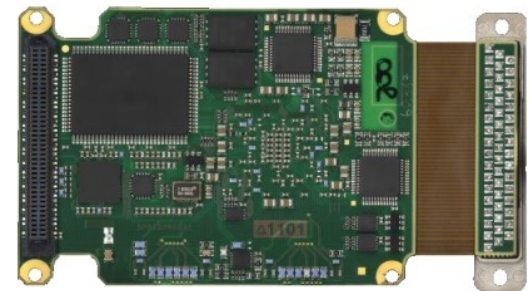

Figure 1: example of rigid/flex PCB technology

Component package: System In Package (SIP) consists of a number of dissimilar integrated circuits enclosed in a single highly miniaturized package. The SIP performs all or most of the functions of an electronic system, and, it can 
contain several silicon components (bare die or package) and passive components. Safran Data Systems has successfully experienced the 3D integration of the versatile analog frontend of ANA module issued from the XMA Digital Acquisition Unit (DAU) widely used in flight test instrumentation. This $3 D$ integration has been conducted using the 3D-Plus technology, one the key player in innovative 3D electronic packaging [5].

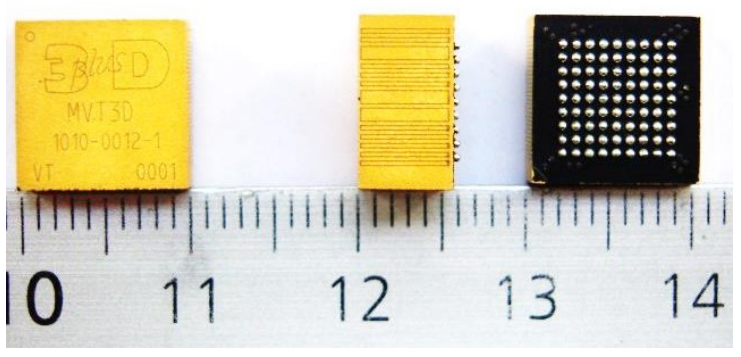

Figure 2: 3D integration of an analog front-end using SIP 3D packaging technology

\section{$\mu$ MA concept}

Inspired by the flight test community feedback and the various trends discussed above, Safran Data Systems has decided to investigate disruptive paths in order to identify and mature the technology bricks needed for a new kind of instrumentation systems. A bank of new ideas have been generated during a set of creativity sessions where design engineers (hardware, software, mechanics, industrialization,...), product owners, sales managers, end-users (test and instrumentation engineers) from the aerospace domain but also from automotive and industry in general, met together to share their return of experience and dream of the future. These sessions were catalyzed by designers and ideation experts in order to stimulate creativity and conceptualize the main ideas.

One axis of the exploration process was about an alternative form factor to the parallelepiped housing. The main drivers of the exploration process, presented hereafter were: ease installation, ease daily operations and minimize intrusiveness.

\section{Ease installation}

All the chassis and stacks on the market requires the instrumentation engineer to design specific mounting plate in order to install the devices in the test vehicle. This step is costly, time consuming and affects the reactivity facing changing conditions. Among other ideas identified to address this pain point, the cylindrical shape appeared to be an interesting alternative. Consequently, it has been decided to baseline the concept on a tube housing. It is a good fit to cylindrical connectors mounted on both ends and allows to use COTS brackets, clamps or straps already used in the vehicle itself (to maintain harnesses and pipes) so already available as in the inventory of the final user. They are affordable, exist in different sizes and materials in order to cope with the local environmental constrains (mainly mechanical and thermal). The Figure 3 shows examples of ideas captured by the designers to illustrate and discuss the various concepts, while the Figure 4 and Figure 5 respectively show the CAD model imagined by the design office and the real device manufactured for the Proof of Concept (PoC) using fast prototyping process.

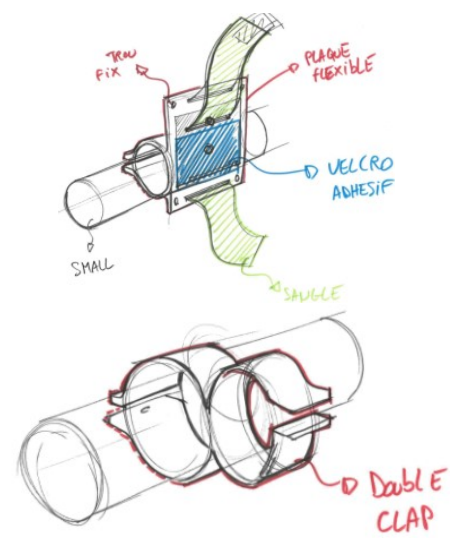

Figure 3: cylindrical shape concept

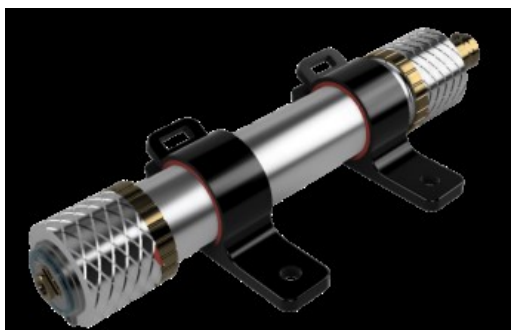

Figure 4: 3D CAD model rendering

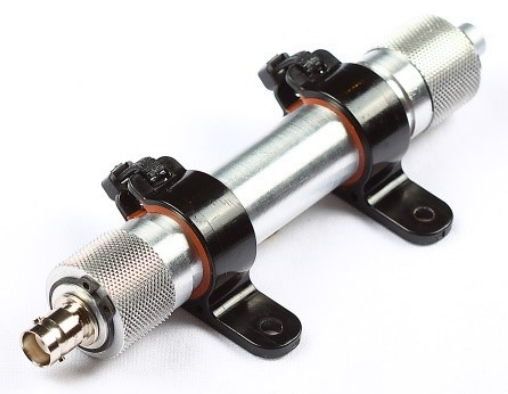

Figure 5: $\mu$ MA Proof of Concept (PoC)

\section{Ease daily operations}

One of the key drivers was to keep the concept modular to offer the instrumentation engineer all 
the versatility expected to cope with unique needs. In this respect, a "building brick" concept, called "cells" has quickly made a consensus among the contributors. The electronics shall be enough packaged to let the cells being manipulated by the final user without any risk of failure. The end-users promoted the fact that the complete assembly/disassembly of the device shall be performed without the need of any tool in order to ease operations on the field. The statement about internal connector discussed previously motivated the team to try to an innovative way to get rid of traditional internal connectors. In the selected concept the internal connectors are replaced by concentric PCB copper patterns to transfer power supply, data communication and synchronization signals. The efficiency of the electrical connection between the cells is insured by the addition of an interposer with anisotropic conductive properties. This interposer is based on an elastomeric material that insures a certain level of tolerance to mechanical axial or angular misalignment. It also acts as a mechanical decoupling interface between all cells which provides a very good behavior facing high level of vibrations and shocks. The concentric pattern is composed of 8 circles, providing a total axis of freedom in terms of relative angular positioning of two adjacent cells. The position and width of each circle is determined according to the nature of the electric signal to be routed but also takes into account the relative positioning uncertainty and the pitch of the conductive elements of the interposer. For the final user, building his own device should not create any headache: the insertion and extraction of the cells are as easy as opening an aspirin tube to get (or insert) a tablet!

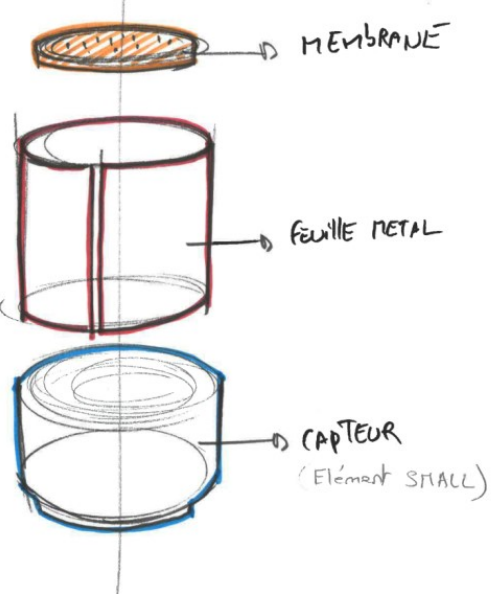

Figure 6: Birth of the cell concept

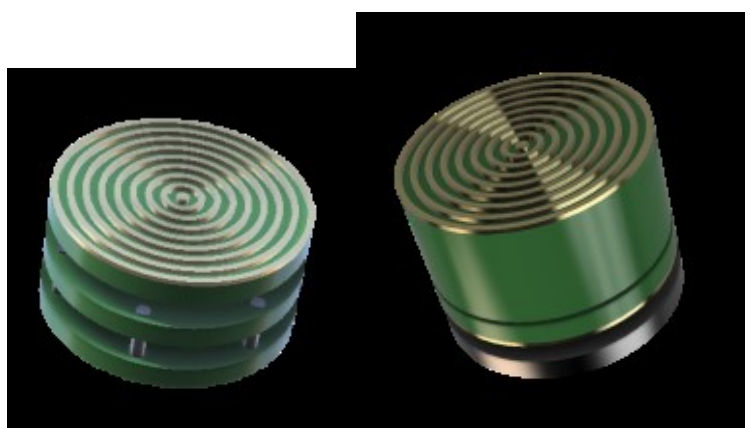

Figure 7: 3D CAD models of the cells

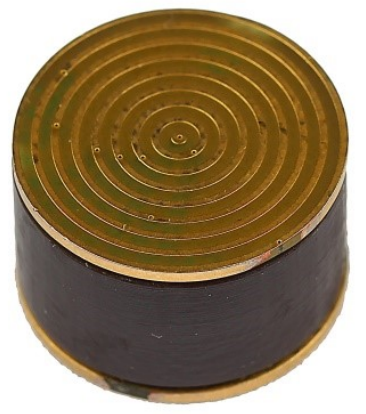

Figure 8: prototype of $\mu M A$ cell

\section{Minimize intrusiveness}

Keeping the device as small as possible was a heavy constrain that everyone kept in mind during all the ideation and design phases. The weight optimization directly inherits from the downsizing effort but also dictated some choices in terms of materials (mechanical housing, potting...). The IloT trend also shows the way to minimize intrusiveness by paying an accurate attention to the power consumption of every single electronic part involved in the design in order to guarantee full power autonomy with also decent life duration. Wireless and less-wire communication technologies have also been selected at early stages of the concept. At the end, the sweet spot between the downscaling willingness and the kind of features needed to be embedded in the device due to integration constraints, led to an optimal diameter being about $20 \mathrm{~mm}$.

\section{From COTS bricks to bespoke solutions}

Once the "cell concept" was agreed among the design team, the next step consisted in identifying the main categories of cells that would compose the device. The challenge here was to define the right level of modularity in order to insure that the concept will be versatile enough to cover the wide range of potential applications, while staying simple to use and open to embrace future needs not yet identified. As a result, four main categories of cells have been identified: 
Acquisition: these cells host the analog signal conditioning front-end (AFE) and the analog to digital converter (ADC). They are dedicated and therefore optimized for each type of sensors.

Data storage: this optional cell can be added in the device when local storage of the acquired data is required. It is the case when the $\mu \mathrm{MA}$ addresses autonomous data-logger use cases but also when it is inserted in a medium to large scale instrumentation systems, especially wireless based systems, in order to secure or optimize the data transfers. Different kinds of data storage media have been considered, the $\mu S D$ format, compatible with the overall form factor of the $\mu \mathrm{MA}$ concept has been selected as a first choice for the density and performances offered by the latest versions available on the market. Its wide adoption by the consumer and industrial domain makes the $\mu S D$ standard a sustainable future proof of choice.

Energy: behind this generic word, various kinds of power sources are anticipated. The first one is obviously the local battery that can be disposable or rechargeable, as small as a button cell, compliant to the standard AA size or even as big as an 18650 form factor if necessary. A local smart power converter integrated in this cell provides the compatibility between various kinds of battery output voltage and deliver the appropriate constant voltage to the other cells. The power lines between cells are designed as a bus that distributes the power in both directions and therefore can allow an external source to power the cells and/or recharge the internal battery. In the scope of the less wire approach, Power over Ethernet (PoE) has been identified as a good candidate to merge data communication, synchronization link and power.

Processing \& Datalink: initially splitted in separate cells categories, the development of the proof of concept prototypes shown that these two features were intimately linked together. The processing is considered as the brain of the device, coordinating all the features hosted by the different cells so creating the overall function needed by the final user. The communications between the other $\mu \mathrm{MA}$, the other devices of the FTI network, a mobile device (smartphone, tablet,...) or a PC are managed by the processing and datalink cell. Wired and wireless communications will require dedicated cells as the physical interfaces are specific (RF antenna, Ethernet, CAN,...). Even if the rule is to implement only one processing and datalink cell per $\mu \mathrm{MA}$, one use case justifies an exception: a wired and a wireless processing and datalink cell can be connected together in order to create a gateway between the wired and the wireless domains (whatever the type of standards: WiFi, BLE, UWB, Ethernet, CAN, USB,...).

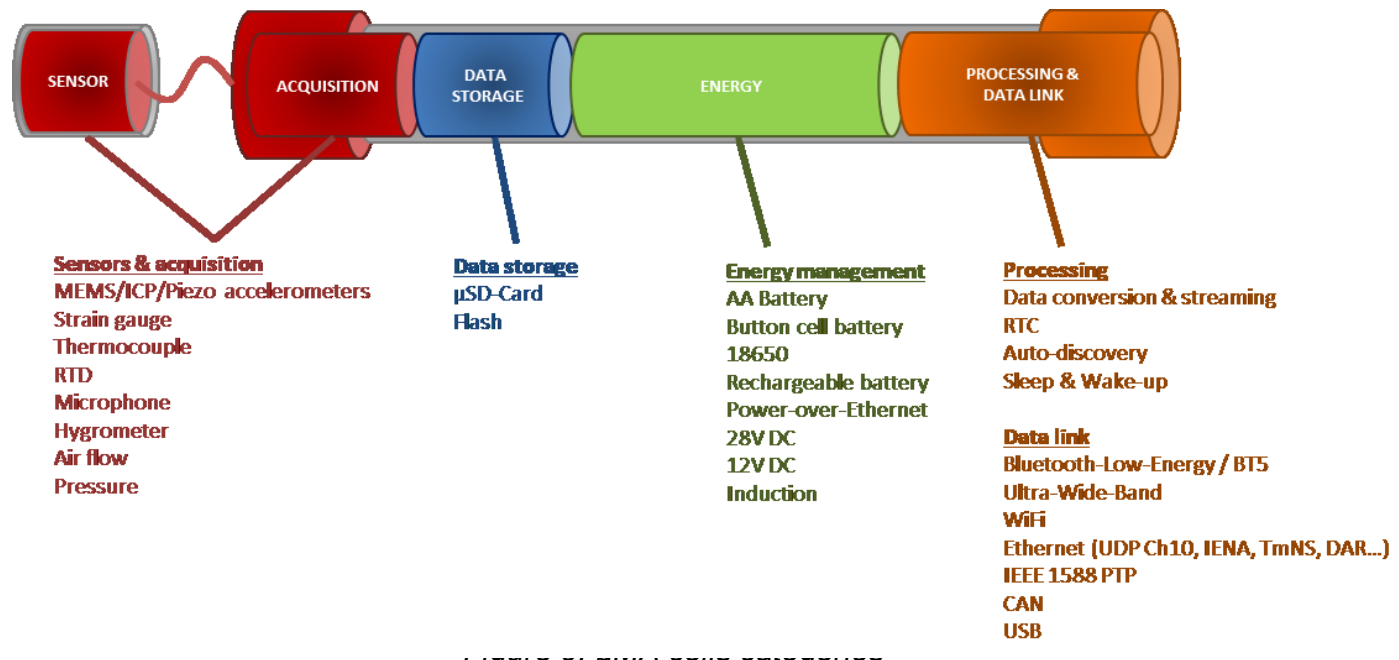

A fifth category of cell has also been added: Sensor. It appears that for some kind of measurements, it was possible to embed the sensing part directly inside a cell, taking benefits of the concept architecture and giving the instrumentation engineer an access to sensors designed to be soldered on a PCB. Ambient air temperature and hygrometry sensors but also MEMS microphones have been identified among others as good candidates for being directly integrated into a cell. This possibility brings also the need to consider the sensing and acquisition cells to be remotely placed from the main part of the device (hosting the battery, the recording, the processing and datalink cells) in order to ease the installation of the sensing part as close as possible to the area or piece to be instrumented 
while digitizing the information as early as possible. In such scenario, the wired connection between the remote part and the main body of the device is composed of the exact same number and type of connection available on the cells themselves.

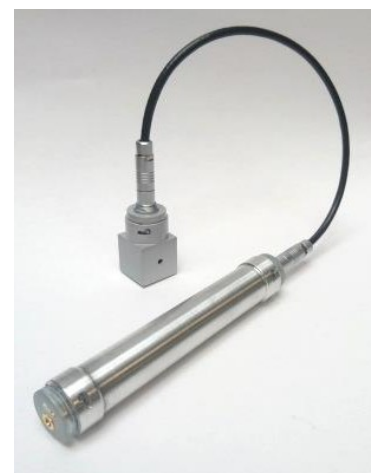

Figure 9: illustration of the concept of $\mu \mathrm{MA}$ remote sensing cell

\section{Auto-discovery \& self-configuration}

Compared to other DAUs on the market that cover wide range of features with a limited set of part numbers thanks to a high level of configurability, the $\mu \mathrm{MA}$ concept is based on very specific cells dedicated to a given sensor or function. It is therefore totally possible to leverage the choice made by the final user at the time of assembly to determine the expected behavior. Being able to understand the users' expectations is a way to optimize the setup phase and even to completely bypass it in some cases. This optimization requires to have an accurate knowledge of the various cells connected together using an auto-discovery and identification process at power-up. This analysis role is assigned to Processing and Datalink cell. The user can select the expected behavior to be applied by default (e.g. acquisition of the maximal input range \& recording; streaming; sleep mode;...). It is of course possible to apply a legacy configuration process to fine tune every settings when needed.

\section{Network Architecture}

From an architectural point of view, it is possible to use the $\mu \mathrm{MA}$ concept independently as a stand-alone device but also connected to others - with or without wires - as light FTI. Extension of an existing FTI (last minute modifications for example) is also a use case.

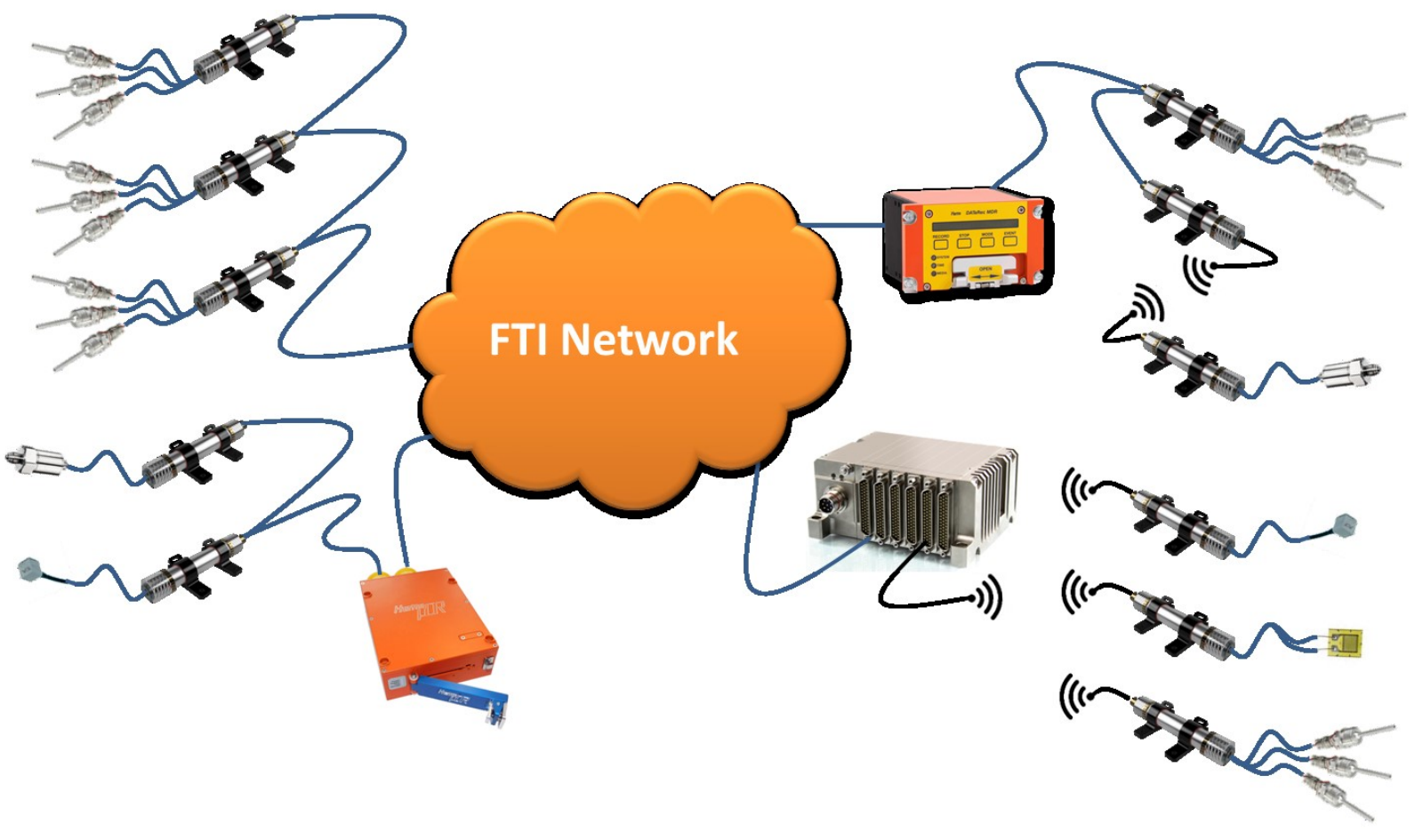

\section{$\mu$ MA concept assessment}

Numerous prototypes have been manufactured in order to evaluate and adapt size, weight, ergonomic aspects, performances, harshness, etc... An agile methodology has been applied to quickly test and validate the different aspects of the concept. Some tests went well on the first run, others failed, and in this case, the design team learned, improved the concept and tried again. The tests started at Safran Data Systems labs, Test \& Training Center and then in real aircraft environments. 
Among these tests, the behavior while facing high levels of vibrations and shocks of the innovative assembly of the $\mu \mathrm{MA}$ concept has been carefully studied in our Vibration Lab. Various ways of mounting the device (including P-clamps) have also been tested. Thanks to the very light weight of the device and the presence of the elastomeric interface between the cells, all the mechanical test campaign was successful.
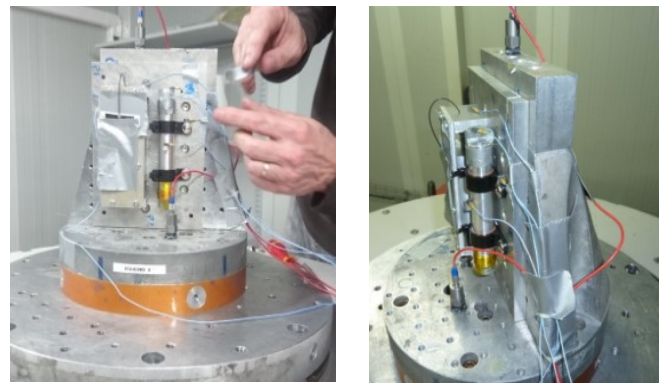

Figure 11: $\mu M A$ prototype during mechanical test campaign

In terms of Electromagnetic compatibility (EMC) derisking tests have been conducted to
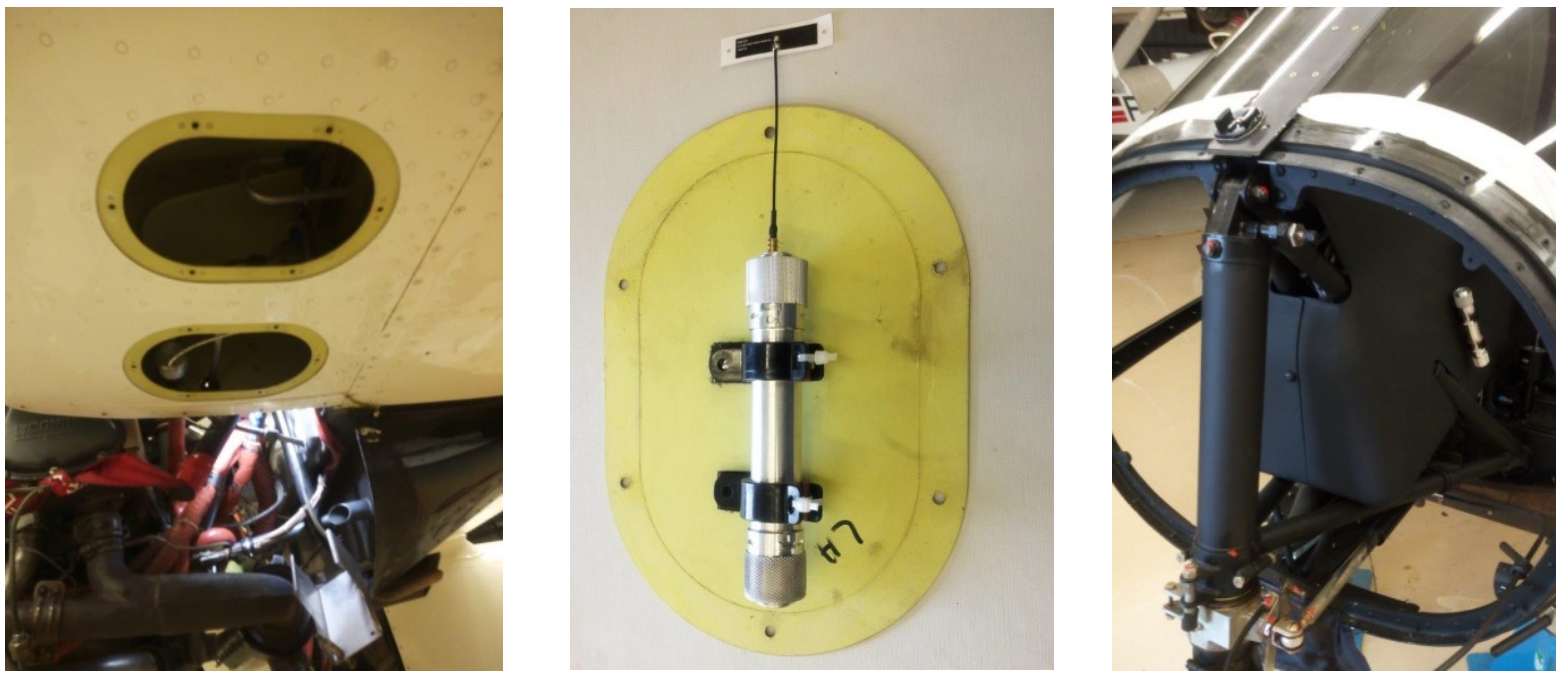

Figure 12: $\mu M A$ prototypes mounted on a Vulcanair P68 aircraft

\section{Conclusion}

Many constraints apply to flight test instrumentation. It must be designed to be integrated into a complex and often tiny structure and to face extreme environments (temperatures, pressures, shocks, vibrations, EMI and EMC, etc.) while targeting to get ever more knowledge about the vehicle. The loT, which uses the same value information loop as the flight test instrumentation, paves the way to a lot of new technologies (e.g. wireless or low power). At the same time, electronics continue evaluate the amount of energy radiated by the device. Thanks to the use of low power components with optimal clock speed the tests were fully passed with radiated levels barely unable to be detected by the measurement tools of the electromagnetic laboratory.

The wireless communication capability of the $\mu \mathrm{MA}$ prototype has been tested with a Bluetooth Low Energy (BLE) implementation. Radio link performance has been tested at various data rates and transmitting power in various environments including in a recent wide body commercial aircraft and also in a twin engine light aircraft as shown on the Figure 12.

All the tests conducted helped to mature the different components of the $\mu \mathrm{MA}$ prototypes and led to the validation of the overall concept. 
as a light FTI with a new type of distributed architecture but also as an extension of an existing FTI (last minute modifications for example).

This success is not trivial. It has required capitalizing on decades of experience in innovation and development of instrumentation systems, imagine new solutions and usages with instrumentation players, thinking of systems and architectures in a new way, to develop a new modular approach of 3D electronics, and to investigate low-power, wireless and energy technologies from the world of IoT and adapt them to the environment of test instrumentation on aircrafts, helicopters, armaments, launch vehicles, naval vehicles, automotive or industrial systems.

Our investigations, our tests in laboratory and in real environments, demonstrate that this new approach of the desing (3D modular electronics, wireless, low consumption, distributed architecture, reduced dimensions, compliance to harsh environmental conditions,...) and usage (instrumentation as close as possible to the sensor, no modification of the structures, high speed deployment, immediate reaction capacity, live troubleshooting, auto-configuration, plug and play, rapid reconfiguration,...) creates a revolution in instrumentation, testing and certification activities for the transportation vehicles of today and tomorrow. This survey is only the very promising start of an adventure towards new use-cases still to be invented for this type of modular platforms.

The overall $\mu \mathrm{MA}$ concept presented in this document is patented and remains the full property of Safran Data Systems.

\section{References}

[1] X. Delarue, "Network telemetry: a new digital acquisition system for Airbus A380", ITC 2004 proceedings, p645-654

[2] G. Garnier, F. Percie du Sert, "Implementing wireless solutions for data transmission and synchronization in the aerospace FTI constraints", ETTC 2019

[3] M. E. Raynor, M. J. Cotteleer, "The more things change: Value creation, value capture, and the Internet of Things" in Deloitte Review, issue 17, 2015.

[4] Multi CB web site as of 05/05/2020 www.multicircuit-boards.eu/en/products/printed-circuitboards/rigid-flex-pcb.html

[5] 3D plus Website System In Package https://www.3d-plus.com/product.php?fam=1

\section{Acronyms}

ADC: Analog to Digital Converter

AFE: Analog Front End

BLE: Bluetooth Low Energy

CAD: Computer Aided Design

CAN: Controller Area Network

COTS: Commercial Off The Shelf

DAU: Digital Acquisition Unit

FTI: Flight Test Instrumentation

HD: High Density

IOT: Internet Of Things

IIOT: Industrial IOT

MEMS: Micro Electro-Mechanical System

PC: Personal Computer

PCB: Printed Circuit Board

PoC: Proof of Concept

PoE: Power over Ethernet

RF: Radio Frequency

SIP: System In Package

SWAP: Size Weight And Power

UAV: Unmanned Aerial Vehicle

USB: Universal Serial Bus

UWB: Ultra Wide Band

$\mu S D$ : micro Secure Digital

All credits: Safran Data Systems 